\title{
Relation between Manual Rotation and Abductive Reasoning in Q-Methodology
}

\author{
Noori Akhtar-Danesh ${ }^{1 *}$, Noeman Mirza ${ }^{2}$ \\ ${ }^{1}$ School of Nursing, McMaster University, Hamilton, Canada \\ ${ }^{2}$ School of Nursing, Thompson Rivers University, Kamloops, Canada \\ Email: ${ }^{\star d a n e s h n @ m c m a s t e r . c a ~}$
}

How to cite this paper: Akhtar-Danesh, N. and Mirza, N. (2017) Relation between Manual Rotation and Abductive Reasoning in Q-Methodology. Open Journal of Social Sciences, 5, 198-204.

https://doi.org/10.4236/jss.2017.53017

Received: February 23, 2017

Accepted: March 21, 2017

Published: March 27, 2017

Copyright $\odot 2017$ by authors and Scientific Research Publishing Inc. This work is licensed under the Creative Commons Attribution-NonCommercial International License (CC BY-NC 4.0). http://creativecommons.org/licenses/by-nc/4.0/ (c) (i) \&) Open Access

\begin{abstract}
Subjectivity is usually evaluated using qualitative research methods. However, Q-methodology offers a different set of techniques for measuring and evaluating subjective viewpoints. Q-methodology is a combination of qualitative and quantitative research techniques that is used to identify unique as well as common viewpoints. The quantitative component of Q-methodology is based on factor analysis and factor rotation. A common approach of analysis in Q-methodology is the use of a centroid factor extraction followed by a manual rotation. Some advocates of manual rotation technique claim that manual rotation is based on the abductive reasoning principle. This article shows that manual rotation and abductive reasoning are two different approaches serving different purposes. Abductive reasoning is a method of hypothesis generation while manual rotation is a method of hypothesis testing. Manual rotation does not conform to abductive reasoning principle if there is no pre-specified theory or hypothesis and consecutive manual rotation of factors toward a satisfactory solution is not the same as rotating factors based on adductive reasoning principle.
\end{abstract}

\section{Keywords}

Q-Methodology, Abductive Reasoning, Manual Rotation

\section{Introduction}

Subjectivity is a complex phenomenon based on individual personal impressions, feelings, and opinions rather than external facts [1]. Its importance is that it represents human perception. Subjectivity is usually evaluated using qualitative research methods. However, Q-methodology offers a different set of techniques for measuring and evaluating subjective viewpoints. It was introduced by William Stephenson [2] [3] and is a valuable tool in exploring human perceptions and interpersonal relationships [4]. In this methodology a combination of 
qualitative and quantitative (statistical) methods are used to uncover different patterns of thought among a group of people rather to identify their numerical distribution. The primary objective of Q-methodology is to identify a typology, not to test the typology's proportional distribution within the larger population [1] [5].

The quantitative component in Q-methodology is based on factor analysis and factor rotation. A common approach of analysis which was adopted by William Stevenson [2] [3] is centroid factor extraction followed by a manual rotation. Although manual rotation is an appropriate choice when there is some predetermined hypothesis or theory, it is not a scientifically sound option when there is no pre-specified hypothesis. In this case, by using a manual rotation the researcher is simply examining the data space for a self-perceived satisfactory solution. Since finding such solution is quite subjective and differs between different researchers, there will be no reliability in the findings. The other approach is using one of the statistical rotation techniques such as varimax, quartimax, etc. There are two schools of thought on whether the factor rotation in Q-methodology should be conducted objectively (using some statistical approach) or subjectively based on manual rotation. In addition, some proponents of manual rotation frequently claim that manual rotation is based on the abductive reasoning approach. The objective of this article is twofold; 1) to critically review the merits of manual rotation and where it might be appropriate, 2) to examine the association between manual rotation and abductive reasoning.

In the following sections we briefly explain the concepts of manual rotation and abductive reasoning. We also present a critical review on the association between these two concepts.

\section{Manual Rotation}

Usually, in the original set of extracted factors, most variables load highly on the first factor and the factors are typically not meaningful or easily interpretable. Factor rotation, as originally explained by Thurstone [6], is a process in which the original factors are rotated about their origin for a simple structure and more interpretability. Tabachnick and Fidell [7] listed ten methods of factor rotation, of which five methods (varimax, quartimax, equamax, promax, and direct oblimin) are implemented in the statistical program of SPSS. The first three are orthogonal and the last two are oblique. On the other hand, manual rotation technique, also known as theoretical rotation, judgmental rotation, or hand rotation, is an orthogonal rotation method that allows researchers to manually rotate the factors on any direction and at any size around the origin. Manual rotation is available only in Q-programs such as PQ Method [8] and PCQ [9]. Although it seems to be an appropriate tool for using with some pre-determined theoretical context, it is used frequently without any pre-defined theory; i.e. the factors are manually rotated until some convincing solution is found which leads to a non-theory based and subjective data-driven solution. This practice is highly subjective because it depends on who is conducting the analysis and whether the 
researcher finds the findings interesting and satisfying. Obviously, based on this practice there will be no reliability in the findings and the results will hardly be reproducible which is in contrast with the principle of scientific query that study findings are required to be reproducible [10]. Stephenson [11] who pioneered use of manual rotation in Q-methodology suggested that for using this technique a researcher should either have a theory, hypothesis, or guess in mind for the factors' positions in the data space. Then, factors are manually rotated to confirm or reject the theory or hypothesis. On the other hand, the common practice in Q-methodology is manually rotating the original factors and evaluating the results until some convincing results are found. The proponents of this practice might claim that it is an exploratory tool that provides the freedom of discovering new factors in the dataset, but they are simply overlooking the exploratory power of mathematical factor rotation techniques (e.g. varimax, quartimax, promax, etc.). The truth of the matter is that all mathematical factor rotations are exploratory and can discover the clusters of similar Q-sorts, i.e. factors, better than any trial and error approach, particularly if there are more than three factors available in the dataset. It is because in manual rotation at each time only two factors can be manually rotated and the data analyst can easily lose sight of other factors that are not being rotated. In addition, position of any Q-sort on any two previously rotated factors will be changed if any of these factors is being re-rotated in conjunction with some other factor.

\section{Abductive Reasoning}

According to Shank [12], historical empirical research aimed to uncover truth by deriving new truths from existing truths based on either observations (e.g., grass is green) or facts that were logically self-evident (e.g., two objects cannot occupy the same space at the same time). The need for a systematic way to discover truths led researchers to adopt deductive reasoning which was built based on Aristotle's syllogism. This allowed researchers to apply one truth to another in order to derive a conclusion which by nature was also true (e.g., if $\mathrm{A}=\mathrm{B}$ and $\mathrm{B}=\mathrm{C}$, then $\mathrm{A}=$ $\mathrm{C})$. Deductive reasoning remained the predominant way of conducting empirical research until the seventeenth century when it was replaced by inductive reasoning by Francis Bacon [12]. Whereas deductive inference allowed specific empirical truths to be derived from general truths, inductive reasoning allowed empirical generalizations to be made from the analysis of specific truths [13].

Inductive reasoning attracted criticism in the nineteenth and twentieth centuries; Karl Popper [14] [15] argued that it is impossible to prove scientific theories as true through inductive reasoning since no amount of evidence guarantees the absence of contrary evidence. He suggested that science can only be accomplished through hypothetico-deductive reasoning. Through this approach, researchers formulated hypothesis based on a collection of facts and tested them with attempts to falsify them [16]. According to Haig [17], hypothetico-deductive reasoning is a restrictive account of method which does not address how theories or hypotheses are created prior to being tested. He argues that the hypothe- 
tico-deductive method is confirmation ally lax, meaning that any positive confirming instance of a hypothesis based on this method "can confirm any hypothesis that is conjoined with the test hypothesis, however plausible, or implausible, that conjunct might be" [p. 372].

Abductive reasoning was introduced by the philosopher C. S. Peirce in the $19^{\text {th }}$ and $20^{\text {th }}$ centuries. It involves reasoning from the phenomena to the theoretical explanation or hypothesis [17]. The difference between abductive reasoning and other forms of reasoning can be explained using the premise (e.g. kidney disease) and the consequence (e.g. increase in creatinine level). Since the premise in deductive reasoning guarantees the consequence as true, a deductivist would argue that:

Patients with kidney disease have increased creatinine levels.

Ken has kidney disease.

Therefore, Ken has increased creatinine level.

In inductive reasoning, the premise provides reasons that are good enough to show that the consequence is probably true. Hence, an inductivist would argue that:

Ken has kidney disease.

Ken has increased creatinine level.

Therefore, patients with kidney disease have increased creatinine levels.

In hypothetico-deductive reasoning, while the premise suggests the consequence, the premise must be tested to determine if, in fact, it provides sufficient reasons to show that the consequence is true. Therefore, a hypothetico-deductivist would argue that:

Ken has increased creatinine level.

Patients with kidney disease have increased creatinine levels.

Therefore, Ken has kidney disease.

In abductive reasoning, however, the premise does not guarantee the consequence, i.e., the relationship between premise and consequence is hypothetical and could be true or false. Thus, an abductivist would argue that:

Patients with kidney disease have increased creatinine levels.

Ken has increased creatinine level.

Therefore, Ken has kidney disease.

These examples aim to demonstrate that hypotheses or theories are generated through abductive reasoning. Therefore, abductive reasoning approach is used for theory or hypothesis generation. Such hypothesis then will be tested through hypothetico-deductive reasoning. This allows for the formation of generalizations (i.e., inductive inference).

\section{Relation between Manual Rotation and Abductive Reasoning}

Before discussing the relation between manual rotation and abductive reasoning 
one point needs to be explained. One common problem in mainstream literature of Q-methodology is the mix-up between centroid factor extraction, manual rotation, and factor indeterminacy [18]. In a nutshell, centroid factor extraction and manual rotation are used interchangeably while they are two different techniques and serve different purposes. Factor indeterminacy is a concept that although loosely defined in Q-methodology, is used as the main reason for using both centroid factor extraction and manual rotation [18].

The issue of connection between abductive reasoning and Q-methodology originated from William Stephenson [11] and advocated by some Q-methodologists including Brown \& Robyn [19]. To justify such connection Stephenson [11] criticized an example by Cattell on the study of temperament [20] where Stephenson claims that Cattell spent six years searching for a simple factor structure. Stephenson argued that his solution based on manual rotation could take only "a few hours along abductory lines". He concluded that "thus, the infinite number of solutions by centroid method [meaning manual rotation] corresponds to the unlimited horizon of abductory methodology" (p. 10). Indeed, what Stephenson was suggesting is that manual rotation provides the researcher the ability to rotate the factors to a position suggested by a specific theory. His main point was that a researcher first needs to have a theory-based position before rotating the factors to that position. His suggestion does not support consecutive use of manual rotation for finding some convincing results. He noted that [p. 13]:

"...the indeterminacy of the centroid solution in factor analysis makes possible the discovery of factors, ..., that is, not as afterthoughts or as a posteriori reasoning, but as abductor-because without the broad abduction or law one wouldn't have known what to look for in the first place".

The main argument in the abovementioned article by Stephenson is that if we have some pre-specified theory, hypothesis, or hunches about a data space of Q-sorts, then we can use manual rotation to examine such theory. In such a scenario, the pre-specified conjecture may or may not be derived abductively but it will be tested using hypothetico-deductive approach. This shows that while manual rotation can be used as a tool to investigate the compatibility of the data with the theory (i.e., using hypothetico-deductive reasoning), there is no other connection between manual rotation and theory generation (i.e., abductive reasoning). Based on this understanding, there is no warranty for findings from a manual rotation to be abductively derived. Not only is this notion contrary to the previously held belief which links manual rotation to abductive reasoning, it has also never been challenged in the Q-methodology literature. Furthermore, it is difficult to claim that the same conclusion can be derived from both manual rotation and abductive reasoning. This is because abductive reasoning is concerned with hypothesis generation while manual rotation aims to evaluate or test a hypothesis.

\section{Discussion}

A common approach of analysis in Q-methodology consists of using a centroid 
factor extraction followed by the manual rotation. In this approach, after factor extraction the researcher inspects the Q-sorts data space in a two-dimensional plane, makes a hypothesis for the position of factors, and manually rotate the factors to that position. This step ends when all the factors are manually rotated. Next, he conducts a Q-analysis of the Q-sorts and examines the distinguishing statements for each factor. If the results do not support the hypothesis, the process is restarted by looking into some other data points and making new hypotheses. This process ends when the researcher is satisfied with the results. This process at the best resembles a hypothetico-deductive approach which is far from an abductory approach that tries to best explain the finding from a mathematical rotation given the available information.

This article shows that manual rotation and abductive reasoning are two different approaches serving two different purposes. Once a pre-specified theory or guess has been generated (whether abductory or not), it can be examined using the manual rotation technique to confirm or reject it. While manual rotation uses hypothetico-deductive reasoning to derive conclusions, they may or may not be consistent with conclusions based on abductive reasoning principle. In conclusion, although manual rotation can be used to examine abductory generated hypothesis, abductive reasoning as a method of hypothesis generation is different from manual rotation and it does not justify the use of manual rotation for consecutive testing of the data space when there is no pre-specified hypothesis.

\section{References}

[1] Akhtar-Danesh, N., Baumann, A. and Cordingley, L. (2008) Q-Methodology in Nursing Research: A Promising Method for the Study of Subjectivity. Western Journal of Nursing Research, 30, 759-773. https://doi.org/10.1177/0193945907312979

[2] Stephenson, W. (1935) Technique of Factor Analysis. Nature, 136, 297. https://doi.org/10.1038/136297b0

[3] Stephenson, W. (1935) Correlating Persons Instead of Tests. Character and Personality, 4, 17-24. https://doi.org/10.1111/j.1467-6494.1935.tb02022.x

[4] Dennis, K.E. (1986) Q-Methodology-Relevance and Application to Nursing Research. Advances in Nursing Science, 8, 6-17. https://doi.org/10.1097/00012272-198604000-00003

[5] Brown, S.R. (1993) A Primer on Q Methodology. Operant Subjectivity, 16, 91-138.

[6] Thurstone, L.L. (1947) Multiple Factor Analysis. University of Chicago, Chicago.

[7] Tabachnick, B.G. and Fidell, L.S. (2001) Using Multivariate Statistics. 4th Edition, Allyn and Bacon, Boston.

[8] PQMethod (2014) Version 2.35, Adapted from Mainframe-Program Qmethod Written by John Atkinson, 1992. Computer Software. http://schmolck.org/qmethod/

[9] Stricklin, M. and Lincoln, N.E. (1996) PCQ: Factor Analysis Program for Q-Technique (Computer Program). Version 3.8.

[10] Houser, J. (2008) Precision, Reliability, and Validity: Essential Elements of Measurement in Nursing Research. Journal for Specialists in Pediatric Nursing, 13, $297-$ 299. https://doi.org/10.1111/j.1744-6155.2008.00171.x 
[11] Stephenson, W. (1961) Scientific Creed-1961: Abductory Principles. The Psychological Record, 11, 9-17.

[12] Shank, G. (1998) The Extraordinary Ordinary Powers of Abductive Reasoning. Theory \& Psychology, 8, 841-860. https://doi.org/10.1177/0959354398086007

[13] Raholm, M.B. (2010) Abductive Reasoning and the Formation of Scientific Knowledge within Nursing Research. Nursing Philosophy, 11, 260-270. https://doi.org/10.1111/j.1466-769X.2010.00457.x

[14] Popper, K.R. (1956) Realism and the Aim of Science. Rowman and Littlefield, Totowa.

[15] Popper, K.R. (1959) The Logic of Scientific Discovery. Basic Books, New York.

[16] Godfrey-Smith, P. (2003) Theory and Reality: An Introduction to the Philosophy of Science. The University of Chicago Press, Chicago. https://doi.org/10.7208/chicago/9780226300610.001.0001

[17] Haig, B.D. (2005) An Abductive Theory of Scientific Method. Psychological Methods, 10, 371-388. https://doi.org/10.1037/1082-989X.10.4.371

[18] Akhtar-Danesh, N. (2016) An Overview of the Statistical Techniques in Q-Methodology: Is There a Better Way of Doing Q-Analysis? Operant Subjectivity: The International Journal of Q-Methodology. (In press)

[19] Brown, S.R. and Robyn, R. (2004) Reserving a Key Place for Reality: Philosophical Foundations of Theoretical Rotation. Operant Subjectivity, 27, 104-124.

[20] Cattell, R.B. (1948) Confirmation and Clarification of Primary Personality Factors. Psychometrika, 12, 197-200. https://doi.org/10.1007/BF02289253

\section{Submit or recommend next manuscript to SCIRP and we will provide best} service for you:

Accepting pre-submission inquiries through Email, Facebook, LinkedIn, Twitter, etc. A wide selection of journals (inclusive of 9 subjects, more than 200 journals)

Providing 24-hour high-quality service

User-friendly online submission system

Fair and swift peer-review system

Efficient typesetting and proofreading procedure

Display of the result of downloads and visits, as well as the number of cited articles

Maximum dissemination of your research work

Submit your manuscript at: http://papersubmission.scirp.org/

Or contact jss@scirp.org 